Infusionstherapie

Transfusionsmedizin

Infusion Therapy Transfusion Medicine

\section{MDS-Patienten profitieren anhaltend von der Behand- lung mit r-HuEPO und G-CSF}

Linz, 6. Februar 1998. Patienten mit myelodysplastischem Syndrom (MDS) profitieren langfristig von der kombinierten Behandlung mit GCSF und rekombinantem humanen Erythropoetin (r-HuEPO). Im Rahmen einer Phase-II-Studie kamen $60 \%$ der transfusionsbedürftigen MDS-Patienten nach der G-CSF/r-HuEPO-Gabe ohne Bluttransfusionen aus. Die Ergebnisse sind so erfolgversprechend, daß eine zur Zeit laufende Phase-III-Studie jetzt den Einfluß auf das Gesamtüberleben klären soll.

Um die bei MDS-Patienten gestörte Proliferation und Ausreifung der erythroiden Vorläuferzellen auszugleichen, bewährt sich offensichtlich die kombinierte Gabe von r-HuEPO plus G-CSF. Ziel der r-HuEPO-Therapie ist es, den Hämatokrit anzuheben und den Transfusionsbedarf zu senken. Aus verschiedenen Untersuchungen weiß man, daß die zusätzliche Gabe von G-CSF die Wirkung von r-HuEPO unterstützt.

In einer multizentrischen Phase-II-Studie der Universitätsklinik Köln wurden 20 MDS-Patienten, zusätzlich zur supportiven Therapie, mit initial 200 I.E. $/ \mathrm{kg}, 3 \times$ pro Woche (s. c.) r-HuEPO plus $1,5 \mu \mathrm{g} / \mathrm{kg} / \mathrm{Tag}$ (s. c.) GCSF behandelt. Zielkriterien der Studie, so Dr. Giuseppe Lentini von der Kölner Universitätsklinik, waren die Verbesserung der hämatologischen Parameter, die Infektionshäufigkeit, das Gesamtüberleben sowie die Frage, in welcher Weise AML-Übergänge beeinflußt werden.

In die Studie wurden Patienten der mittleren Risikostufe (intermediate risk I/II) aufgenommen (Karnofsky-Index $>60 \%, \mathrm{Hb} \leq 9 \mathrm{~g} / \mathrm{dl}$, Neutrophile $<3 \times 10^{3} / \mu$ l, Thrombozyten $<100000 / \mu l$ ), die meisten MDS-Patienten waren transfusionsabhängig. 15 der 20 Patienten sprachen auf die Behandlung in dem Sinne an, daß entweder die Transfusionshäufigkeit um mindestens $50 \%$ reduziert wurde oder sich der $\mathrm{Hb}$-Wert um mindestens 1 $\mathrm{g} / \mathrm{dl}$ erhöhte. «Insbesondere unter Berücksichtigung der Risikokonstellation ist die Ansprechrate von 75\% ein sehr gutes Ergebnis», betonte Lentini. Angesichts des nicht unbedeutenden Risikos einer Bluttransfusion bezeichnete er es als sehr erfreulich, daß ungefähr $60 \%$ der initial transfusionsbedürftigen Patienten keine Bluttransfusion mehr benötigten. Am besten sprachen die RA- und die RARS-Patienten auf die Behandlung an

Einen Neutrophilenanstieg (>3000/ $\mu$ l) erzielten 18 der 20 Patienten (90\%). Die Therapie hatte keinen stimulierenden Einfluß auf die Thrombozyten. Erfreulich war auch die gute Verträglichkeit der kombinierten Gabe von r-HuEPO plus G-CSF.

Große Bedeutung mißt Lentini den guten Ergebnissen im Langzeitverlauf zu. Nach einer Therapiedauer von im Median immerhin knapp 54 Wochen (maximal 164 Wochen) blieb der mit $85 \%$ weitaus größte Anteil der Patienten FAB-Subtyp-stabil. Es kam also bei diesen Patienten zu keinem Anstieg der Blasten. Nur 15\% der Patienten machten einen Progreß durch: Meist handelte es sich um Übergänge in eine RAEB. Bei einem Patienten kam es zu einem AML-Übergang, an dessen Folgen er dann auch verstarb.

Für Lentini steht fest, daß die kombinierte Behandlung mit r-HuEPO plus G-CSF eine wirksame Therapie für MDS-Patienten mit mittlerem Risikoprofil ist. Von besonderer prognostischer Bedeutung ist die offensichtlich anhaltende Verbesserung der hämatologischen Parameter. Aufgrund der vielversprechenden Ergebnisse wurde eine Phase-III-Studie initiiert, die den Einfluß auf die Gesamtüberlebenszeit evaluieren wird. Diese Ergebnisse liegen allerdings, so Lentini, noch nicht vor.

Weitere Informationen erhalten Sie bei:

Fleishman-Hillard Germany GmbH

International Communications

Horst Lehmann, Dr. Rolf Hömke

Hanauer Landstraße 182c

60314 Frankfurt/M

Telefon 069 / 4057 02-13

Fax 069 / 430373 